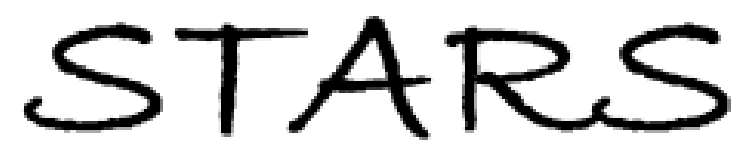

University of Central Florida

STARS

$1-1-2005$

\title{
Characterization of digital-micromirror device-based infrared scene projector
}

\author{
William R. Folks \\ University of Central Florida \\ José Manuel López-Alonso \\ Brian Monacelli \\ Arthur Weeks
}

Guy Zummo

See next page for additional authors

Find similar works at: https://stars.library.ucf.edu/facultybib2000

University of Central Florida Libraries http://library.ucf.edu

This Article is brought to you for free and open access by the Faculty Bibliography at STARS. It has been accepted for inclusion in Faculty Bibliography 2000s by an authorized administrator of STARS. For more information, please contactSTARS@ucf.edu.

\section{Recommended Citation}

Folks, William R.; López-Alonso, José Manuel; Monacelli, Brian; Weeks, Arthur; Zummo, Guy; Mullally, Daniel; and Boreman, Glenn D., "Characterization of digital-micromirror device-based infrared scene projector" (2005). Faculty Bibliography 2000s. 5188.

https://stars.library.ucf.edu/facultybib2000/5188

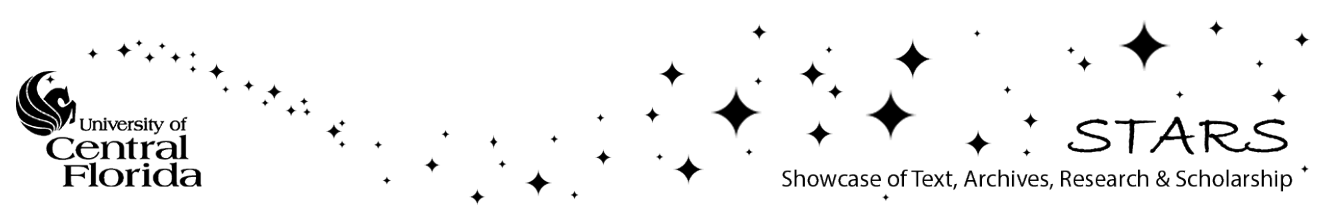




\section{Authors}

William R. Folks, José Manuel López-Alonso, Brian Monacelli, Arthur Weeks, Guy Zummo, Daniel Mullally, and Glenn D. Boreman 


\section{Characterization of digital-micromirror device-based infrared scene projector}

\author{
William R. Folks \\ University of Central Florida \\ CREOL and FPCE College of Optics \\ and Photonics \\ Orlando, Florida 32816-2700
}

José Manuel López-Alonso

University Complutense of Madrid

School of Optics

Avda. Arcos del Jalón s/n

Madrid 28037, Spain

\author{
Brian Monacelli, MEMBER SPIE \\ Arthur Weeks, MEMBER SPIE \\ Guy Zummo \\ Daniel Mullally, MEMBER SPIE \\ Glenn D. Boreman, FELLOW SPIE \\ University of Central Florida \\ CREOL and FPCE, College of Optics \\ and Photonics \\ Orlando, Florida 32816-2700 \\ E-mail: boreman@creol.ucf.edu
}

\begin{abstract}
A test procedure is developed for an infrared laser scene projector, and applied to a projection system that we develop based on digital micromirror technology. The intended use will be for simulation and target training. Resolution and noise are significant parameters for target perception models of infrared imaging systems. System resolution is normally measured as the modulation transfer function (MTF), and its noise modeled through an appropriate signal standard deviation metric. We compare MTF measurements for both mid-wave (MWIR) and longwave IR (LWIR) bands for an infrared laser scene projector based on the digital micromirror device (DMD). Moreover, we use two complimentary models to characterize imaging camera noise. This provides a quantitative image-quality criterion of system performance. () 2005 Society of PhotoOptical Instrumentation Engineers. [DOI: 10.1117/1.2013249]
\end{abstract}

Subject terms: scene generation; infrared scene projection; spatial light modulator.

Paper 040388R received Jun. 18, 2004; revised manuscript received Feb. 15, 2005; accepted for publication Feb. 17, 2005; published online Aug. 29, 2005.

\section{Introduction}

\subsection{Basics}

In comparison with hardware-in-the-loop (HWIL) projectors, scene generation in the infrared has several additional unique requirements for use in simulation and training. ${ }^{1}$ The need to project imagery onto a diffuse reflecting screen viewable from a wide range of angles necessitates a laserbased approach rather than the usual HWIL solution of a resistive array to satisfy radiometric requirements.

The goal of this work is to develop a test procedure to characterize digital micromirror device (DMD) performance due to their low cost and promising applications. The method must be able to quantify the various artifacts that appear during alignment, changes in configuration, unintended electronic noise, screen characteristics, etc. Image quality performance that simulates a realistic imager is desirable for simulation and training purposes. From this point of view, two figures of merit relevant to image quality are discussed. The first is resolution, measured by the modulation transfer function (MTF) of the system and discussed in Sec. 2. The second is noise-related image quality, characterized by a variety of spatial and temporal effects, and discussed in Sec. 3. The advantage to this approach is that both MTF and noise can be divided into component parts to study each subsystem MTF or contributing noise effect.

\subsection{System Characteristics}

Our infrared projection system is based on a reflective spatial light modulator, the Texas Instruments Digital Micromirror Device $\left(\mathrm{DMD}^{\mathrm{TM}}\right){ }^{2}$, which can operate in both midwave (MWIR, 3 to $5 \mu \mathrm{m}$ ) and long-wave infrared (LWIR, 8 to $12 \mu \mathrm{m})$ bands. Our DMD was retrofitted with a $\mathrm{ZnSe}$ window by Optical Sciences Corporation ${ }^{3,15}$ to increase throughput in these bands. The DMD used in this study has $600 \times 848$ tiltable mirror pixels. Each pixel has binary on and off states and a pixel pitch of $17 \mu \mathrm{m}$, which rotates to \pm 10 -deg states. It may be thought of as an actively driven blazed grating. The DMD is driven in binary format at a frame rate of $4065 \mathrm{~Hz}$ to achieve gray levels. The recording camera frame rate is $60 \mathrm{~Hz}$ for our application. For IR scene projection applications, the main performance issue using the DMD is that the pixel size is comparable to the IR wavelength of the source. ${ }^{4}$ For this reason, we investigate the system's modulation transfer function (MTF) using both MWIR and LWIR laser sources.

Two different lasers are used as the source, a $\mathrm{CO}_{2}$ laser at $10.6 \mu \mathrm{m}$, and a $\mathrm{HeNe}$ laser at $3.39 \mu \mathrm{m}$. Figure 1 shows a typical experimental setup with one laser in the system. The beams were spatially filtered and expanded before illuminating the DMD. The result is a 2-D set of diffraction orders. The input angles for the two lasers were adjusted so that the first diffracted order $\left(n_{-1,0}\right)$ of each band was collected and imaged with the screen normal to the optical axis of the lens-DMD combination. In principle, any one of the orders could be used, limited only by the geometry of the setup. The first order provided a convenient in-plane con-

0091-3286/2005/\$22.00 @ 2005 SPIE 

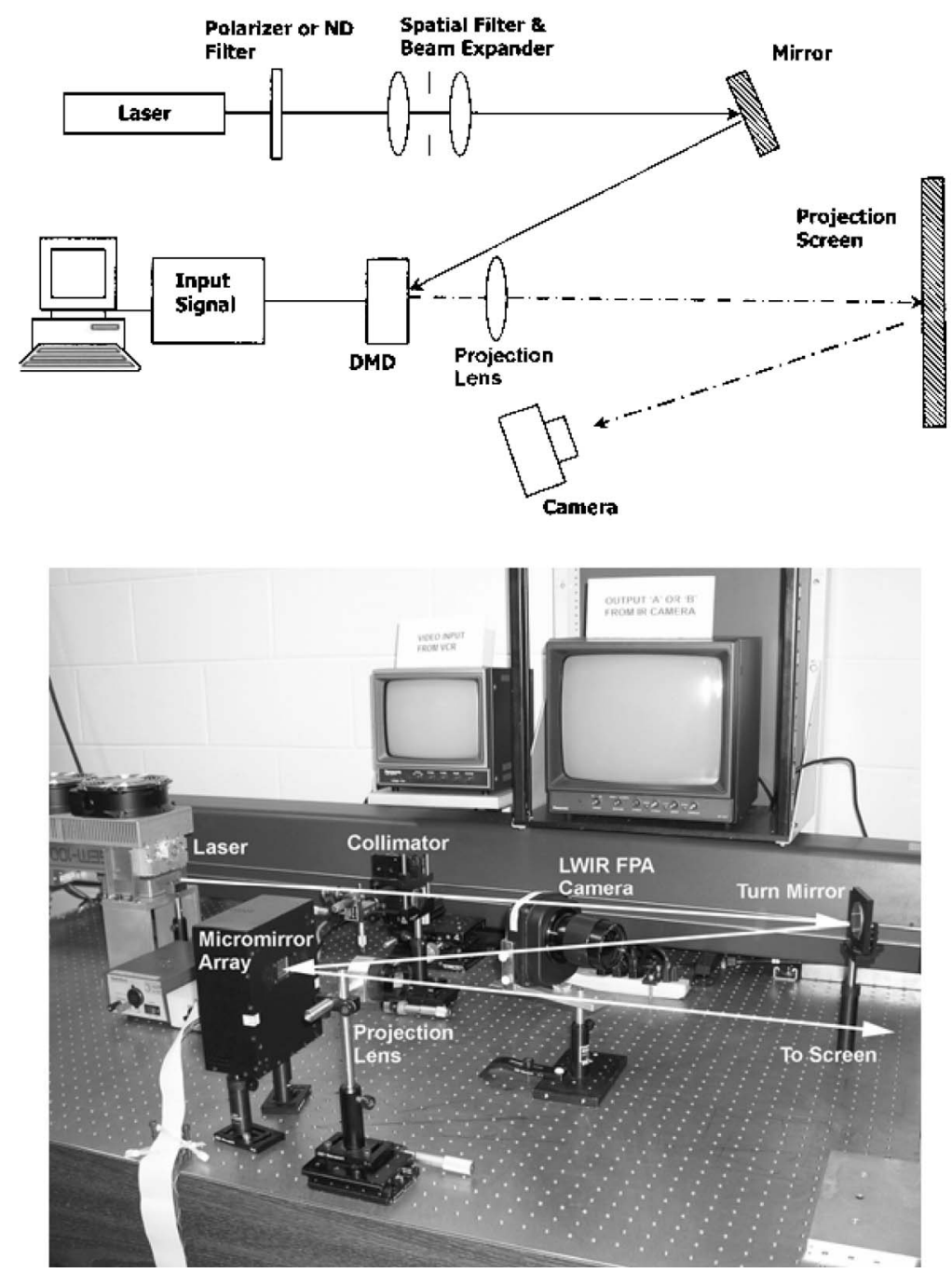

Fig. 1 Typical experimental setup. The FPA camera is focused onto the plane of the projection screen.

figuration. Radiation from the collected order was imaged onto a projection screen by a well-corrected dual-band MWIR/LWIR projection lens with focal length $f=10 \mathrm{~cm}$; aperture $=4 \mathrm{~cm}$; and typically lens to DMD distance $=(11$ to 12$) \mathrm{cm}$, lens scatter screen distance $\cong 100 \mathrm{~cm}$, magnification $\cong 10 \times$, and screen to camera distance $=50 \mathrm{~cm}$. We use a sandblasted $215 \times 280-\mathrm{mm}$ aluminum screen, which has a diffuse reflectivity greater than $90 \%$ in the IR. The image on the screen was viewed with a LWIR [ferroelectric focal plane array (FPA)] or a MWIR (PtSi) camera. For the measurements contained here, the 10.6 - $\mu \mathrm{m}$ laser produced about a $10-\mathrm{W}$ output beam. The 3.39 $-\mu \mathrm{m}$ laser used had a maximum beam power of $40 \mathrm{~mW}$. Power was kept below $10 \mathrm{~W} / \mathrm{cm}^{2}$ to avoid damaging the array. Projection on a larger screen would be possible at higher laser output powers, with proper attention to heat removal at the DMD.

"Off" radiation of the DMD pixels, particularly for the wide diffraction spread in the LWIR, is a potentially significant source of reduced contrast ratio. Optical Sciences Corporation reports a mid-wave IR contrast ratio of 90:1 in their literature, ${ }^{3}$ which is significantly above our measured result reported in Sec. 2. This can expected, as our prototype system was assembled with off-the-shelf components and not optimized for a particular IR band. Further work will address issues of "off" radiation, increased pixel tilt, optimization of optical layout, materials, and image recording.

\section{Modulation Transfer Function Measurements}

Modulation transfer function (MTF) is defined as the modulus of the complex optical transfer function (OTF) and is a convenient figure of merit used to measure system image quality. ${ }^{5}$ It may also be defined as the absolute value of the Fourier transform of the point spread function (PSF),

$\mathrm{MTF}=|\mathrm{F}(P S F)|$

The advantage of the MTF approach is that the total system MTF is simply expressed as the product of each MTF of the subsystems: 


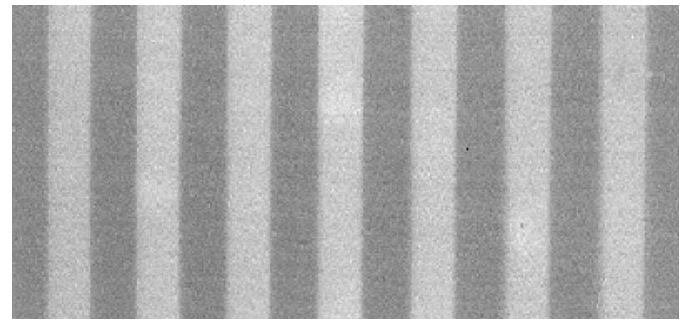

Fig. 2 Typical bar chart pattern captured by the IR camera reflected from a diffuse screen.

$$
\mathrm{MTF}_{\mathrm{system}}=\prod_{i=1}^{n} \mathrm{MTF}_{i}
$$

This property allows for the MTF of each subsystem to be studied independently. In this work, we measured the MTF of the MWIR and LWIR cameras separately from the MTF of the scene projection system, using transparency bartargets with square-wave emissivity ${ }^{6}$ placed at the projection screen. This allowed us to divide out the MTF of the infrared cameras from the data, leaving just the MTF of the scene projection system (DMD and projection lens). We used the approach of a square-wave dataset, ${ }^{7}$ measuring MTF as the magnitude of the fundamental component, ${ }^{8,9}$ as a function of the spatial frequency of the bars. This avoided the necessity of a series correction to convert square-wave to sine-wave data.

A line-by-line series of horizontal slices were extracted from the images of Fig. 2, and the absolute value of the Fourier transform of each line scan is taken and averaged. This procedure is repeated for each frequency of interest. To avoid nonlinearity in the camera responses, square-wave datasets with $40 \%$ modulation depth were used at the DMD input.

As can be seen from Fig. 3, the scene projector had better MTF performance when used at MWIR than at LWIR. The spatial frequency is observed at the projection screen. The MTF of the scene projector, especially for LWIR illumination, drops off more rapidly because of diffraction at the DMD pixels and because the projection

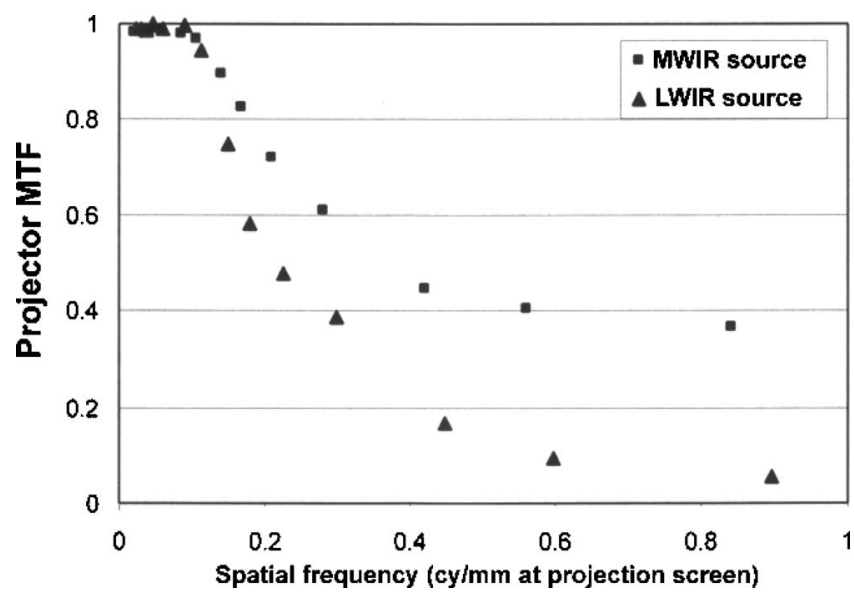

Fig. 3 MTF of the scene projector (with the camera MTF divided out) for MWIR and LWIR sources. lenses were well corrected but not optimized for this optical system. The measured low-frequency contrast ratio of the imagery in LWIR was quite low, about 12:1, mainly because the projection screen itself was a room-temperature gray body at about $10 \%$ emissivity. For MWIR illumination, the low-frequency contrast ratio increased to 25:1. Both curves appear to be flattening out at high spatial frequency. We suspect this is an artifact of our measurement or processing, and would rather expect MTF to roll off monotonically to zero at high frequencies. From the geometry described in Sec. 1.2, the 848 columns of $17-\mu \mathrm{m}$ spaced mirrors can generate a maximum of 35 cycles $/ \mathrm{mm}$ on the display. With magnification $m \cong 10$, the maximum spatial frequency at the screen would thus be $\sim 3$ cycles $/ \mathrm{mm}$, so we are not limited by device electronics. Remember that the images are recorded in reflection off-axis from a textured screen rather than in a hardwarein-the-loop configuration. Possible causes are: 1. diffraction effects, 2. speckle, 3. time averaging, and 4. aliasing effects. For example, we time average over 16 frames for MTF images. This will have a greater relative effect in skewing the result at higher spatial frequencies. Noise measurement is an important analysis tool discussed in the next section, which may help identify additional contributing factors.

\section{Noise Measurement}

\subsection{Noise Models}

Noise in focal plane arrays (FPAs) can be quite complex. A simple description such as the rms value of a reference signal is often insufficient. The readout electronics and FPA manufacturing methods, for example, may introduce correlations among frames, columns, and rows. Several models have been developed to deal with these complex patterns. In this work, we have chosen to apply two complementary models. The first is a $3-\mathrm{D}$ noise model $^{10}$ developed by Night Vision and Electronic Sensor Directorate (NVESD) (Fort Belvoir, VA). An alternative method is a statistical noise model based on a principal component approach (PCA). ${ }^{11,12}$ Both models take a sequence of images from a uniform background as their starting point for noise measurement. In the case of the 3-D noise model, the complete sequence of frames are arranged in a cubic dataset. Inside this cube, it is possible to move in three independent dimensions: horizontal $(h)$, vertical $(v)$, and temporal $(t)$. The horizontal and vertical directions represent the observation plane. A sequence of frames is then collected over time to study the temporal noise behavior. The rms values are calculated depending on the direction within this cube of data. For example, $\sigma_{t v}$ is the rms value when moving in the temporal, horizontal, and vertical direction [vector $(1,1,1)$ ] in the dataset cube after removing any other type of noise. It is related to the temporal noise of the detectors. The set of rms values $\sigma_{v h}$ represents the case of a single frame with the temporal dimension removed, and so on. Frame averaging can be utilized in this last operation. Clearly, this noise is related to nonuniformity of the focal plane array. Other types of 3-D noise parameters can be correlated with physical sources in a similar way. In total, there are eight types of such noises parameters. For a complete review, the reader may consult Refs. 10 or 13. 


\subsection{Principle Component Analysis}

The PCA method also deals with a sequence of frames. However, each frame is taken to be a random event. If $N$ frames are recorded, the total sequence is dealt with as an $N$-dimensional random vector consisting of $N$ random variables ( $N=$ the number of frames). This model analyzes the correlation between frames by means of the correlation between these random variables. The point of departure in this method is the covariance matrix $\mathbf{S}$, which describes the covariance structure among the frames in the dataset. Sequential frames normally exhibit a non-negligible degree of correlation among them. The principal component decomposition method deals with a noncorrelated version of them. Principal components are linear combinations of the original frames with no correlation among them. The challenge is to find the coefficients of these linear combinations. It can be demonstrated that these coefficients are given by the eigenvectors of the covariance matrix $\mathbf{S}$. The problem is then solved by diagonalization of the covariance matrix. Formally, if we note the frames as $F_{t}$, the principal component $Y_{\alpha}$ can be expressed as:

$Y_{\alpha}=\sum_{t=1}^{N} e_{t, \alpha} F_{t}$,

$e_{t, \alpha}$ being the $t$ component of the eigenvector $\alpha$. Equation (3) can be inverted to see the original frames as produced by the principal components,

$$
F_{t}=\sum_{\alpha=1}^{N} e_{t, \alpha} Y_{\alpha} .
$$

The dataset can then be reconstructed with only a specific set of principal components. Various groups of principal components represent different spatial-temporal noise structures ${ }^{10}$ within the entire set. Principal components are like "eigen-images," giving information not only of rms values, but in addition, the spatial-temporal distribution of these rms values. This allows us to break down the noise into its various types, and isolate and study each of them. Such an approach has the advantage of being able to isolate the fundamental causes of noise within a complex optical system and optimize each type of noise systematically. An important feature of the PCA method is that the principal components are obtained with a decreasing order of relevance in the data. This is parameterized by:

$$
\Omega_{\alpha}=\frac{\lambda_{\alpha}}{\sum_{\alpha=1}^{N} \lambda_{\alpha}},
$$

where $\lambda_{\alpha}$ is the eigenvalue of matrix $\mathbf{S}$ corresponding to principal component. From this point of view, each principal component represents a portion of the total variance dataset given by Eq. (5). Two or more principal components may be grouped together into a "noise process." 10 This grouping is made by studying the uncertainties in $\lambda_{\alpha}$. Two principal components represent the same amount of variance when their eigenvalues overlap within uncertainty. A noise process is formed by consecutive overlapping prin-
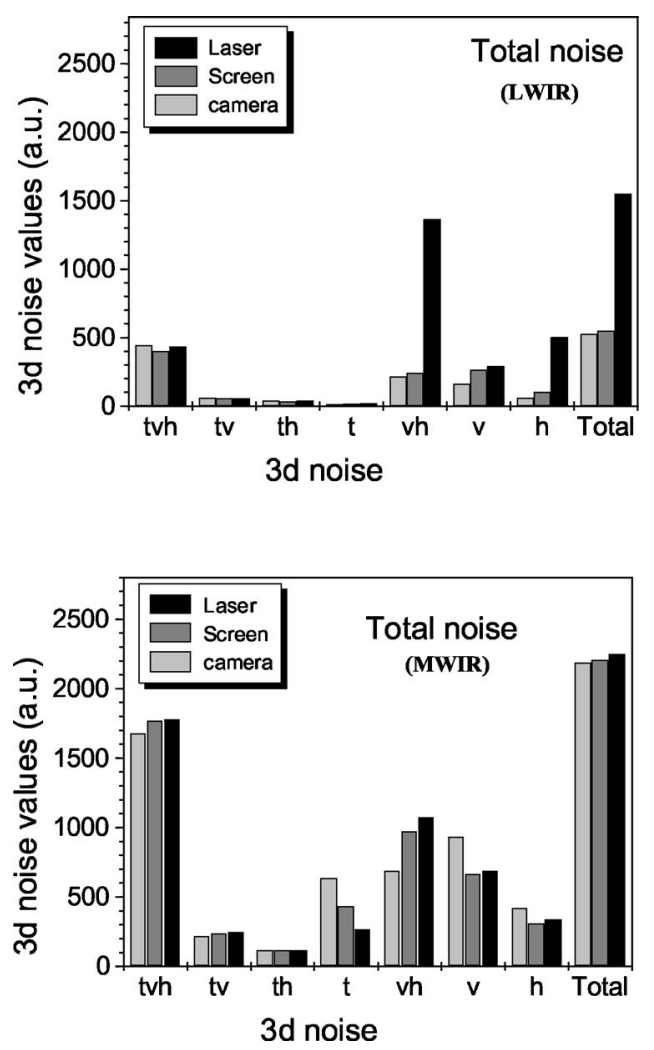

Fig. 4 Results for the 3-D noise model. LWIR camera (upper) and MWIR camera (lower). Noise of three independent dimensions are reported: horizontal $(h)$, vertical $(v)$, and temporal $(t)$.

cipal components. New frame datasets may be formed by reconstructing the original sequence, taking into account different system noise processes. Different imaging systems have been analyzed in this way. ${ }^{12,14}$

\subsection{Measurements With Thermal Cameras}

We apply the noise analysis methods discussed previously to uniform images taken by two different thermal cameras. The MWIR camera is a Mitsubishi model IR-5120A with a PtSi detector. The LWIR camera is a Raytheon Control IR 2000B ferroelectric FPA. Three different types of data are collected, each consist of 50 frames taken at a frame rate of $60 \mathrm{~Hz}$. The first dataset is of the camera with a uniform dark input. The second is taken with the camera focused on the screen and the laser off. The third is with a mid-level uniform gray input image to the projector and the laser turned on.

Results for the 3-D noise model can be seen in Fig. 4. The noise for the LWIR camera is similar for the dark camera and the camera-screen combination. It increases only slightly with introduction of the screen. After the laser is switched on, however, the total amount of noise increases significantly. The increased spatial noise $\sigma_{v h}$ is most noticeable. It couples with other types of system noise, but not temporal noise. On the contrary, the MWIR noise is similar in all three instances. However, there is a minimal increase in spatial noise with the laser switched on.

Next, the PCA method was applied. We expected to see different types of noise when comparing the dark camera, 


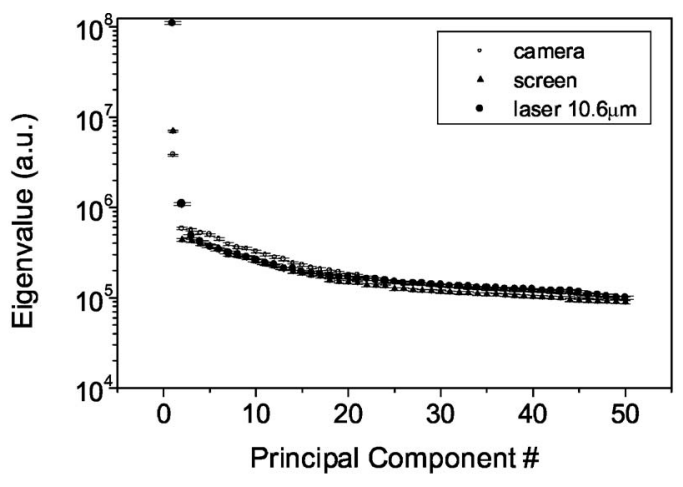

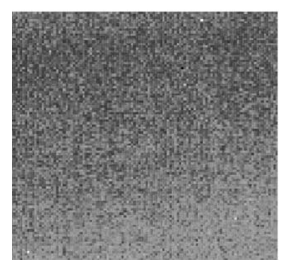

(a)

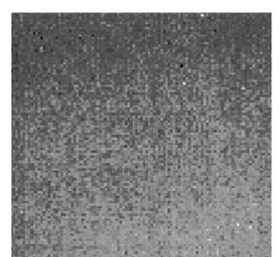

(b)

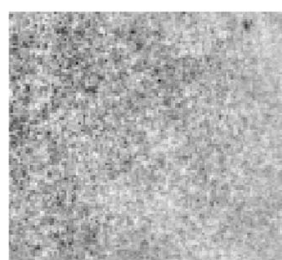

(c)
Fig. 5 PCA results for LWIR camera. Note the increase in spatial noise (first principal component) in the case of laser illumination (upper). The lower eigen-images are of the first principal component appearing in LWIR for (a) the camera itself, (b) looking at the screen, and (c) the screen illuminated with laser. The images are in false scale to enhance spatial structure.

camera-screen, and camera-screen-laser cases. Figure 5 shows the result for the noise processes observed by the LWIR camera. The noise structure for the dark camera and the camera-screen case is rather similar. The value of the first principal component of corresponding spatial noise is higher when the screen is introduced. The major difference in the dataset occurs when laser illumination is applied. Here, the spatial noise has increased and a second isolated process appears. An eigenimage of the first principal component for each case is shown in Fig. 5. It is possible to see similarities in the structure of the spatial noise when comparing data from the dark camera to the camera-screen setup. The increase in spatial noise with the addition of the screen is due to angular dependence of reflection over the field of view, and the fact that the screen in our setup is neither normal to the optic axis, nor a perfect Lambertian reflector. The spatial noise introduced by the laser has a structure resembling speckle and represents the majority of noise in the dataset. A secondary process of interest is shown in Fig. 6, where we see fringe structures that commonly appear as diffraction patterns during alignment (see lower-right corner of image). The time evolution of this pattern suggests cycles of around 2 to $3 \mathrm{~Hz}$, which may be related to laser stability.

The results given by the PCA method for the MWIR camera are shown in Fig. 7. It can be seen that no significant new noise processes appear in the three datasets. The structure of the noise is always a single component representing spatial noise and a "temporal process" consisting of 49 principal components. The first principal component in-
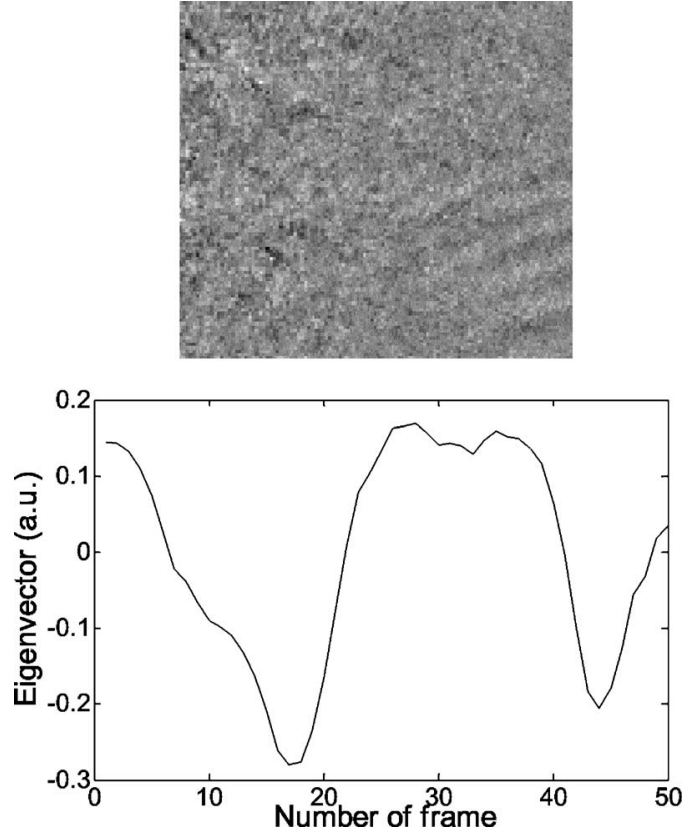

Fig. 6 View of the second principal component appearing in LWIR when illuminating the screen with laser (upper) and its temporal evolution (lower).

side this temporal group appears isolated in the three datasets. Their spatial structure consists of well-defined fringes crossing the field of view. This type of noise is quite common in focal plane arrays ${ }^{10}$ and is related to the electronics of the video signal. Figure 7 illustrates the first principle component of the spatial noise for the three datasets. The rms values of all three are similar, but their spatial structure is different. The change from the dark camera to the camera-screen configuration is again explained by a difference in reflection across the field of view due to off-axis camera orientation. The laser introduces some fringes due to diffraction patterns in the alignment, but there is no influence of speckle patterns over spatial noise. This behavior was expected due to difference in wavelength between the lasers.

To reduce the influence of speckle over the image in LWIR, we have introduced a different type of screen. It consists of a metallic screen with a greater amount of roughness over the surface. We have recorded three different types of data with the LWIR: a uniform dark background with the camera, the camera looking at the new screen without laser illumination, and with laser illumination. Figure 8 shows the results of 3-D noise measurement and first principal component eigen-images for all cases. There is still more spatial noise under laser illumination conditions, but the amount has been considerably reduced when using the new rough screen. [Please note that the scale of the eigen-images are different in Figs. 5(c) and 8(c) although they may appear similar because of the false scale used to enhance spatial structure.] Screen optimization is a matter for further research, taking into consideration not only noise but resolution and system radiometry. 

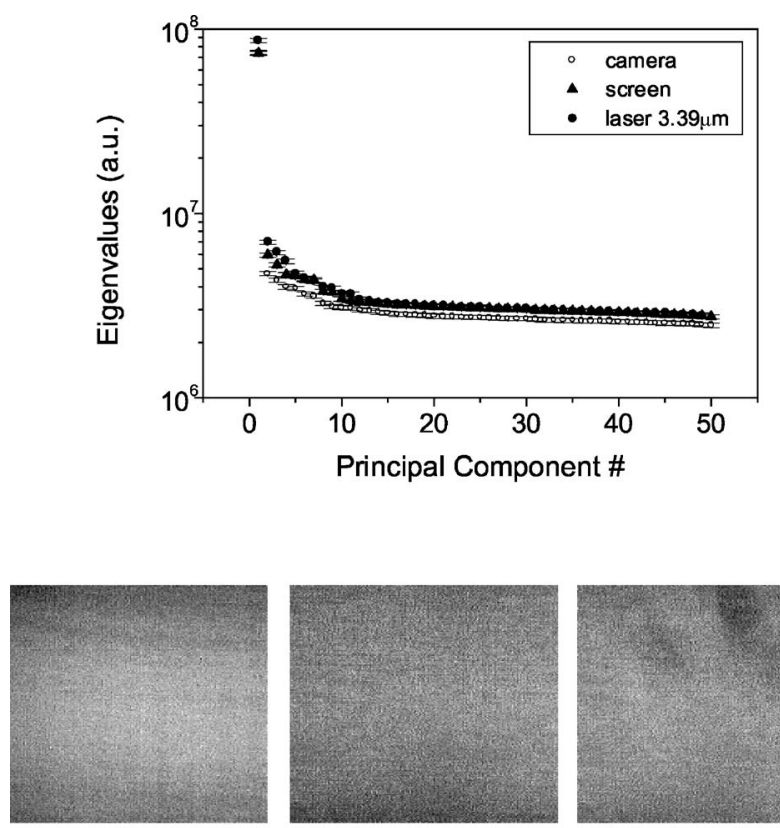

(a)

(b)

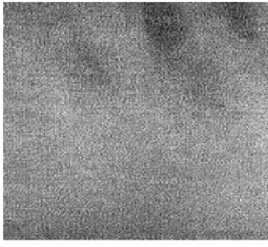

(c)

Fig. 7 PCA results for MWIR camera. In this case, the importance of the different types of noise is the same, independent of the experimental setup used to produce a uniform background (upper). The lower eigen-images are of the first principal component appearing in MWIR for (a) camera itself, (b) looking at the screen, and (c) illuminated with laser. The images are in false scale to enhance spatial structure. The level of rms noise in all images is similar (compared with Fig. 8), but the spatial structure is slightly different, revealing artifacts that depend on the experimental setup used to produce the uniform background.

\section{Conclusion}

We develop an experimental setup to test the performance of a DMD-based infrared scene projector in target perception for training purposes. The method is based on analysis of noise measurements and resolution in LWIR and MWIR. This method measures the scene projector MTF and noise structures occurring for different experimental configurations. Our method can characterize the influence of different experimental setup parameters such as alignment and effects of laser speckle. As expected, MTF performance of the projector was superior for MWIR than for LWIR radiation. In the LWIR, the $17-\mu \mathrm{m}$ pixel size of the DMD is only slightly larger than the wavelength of the illuminating radiation. Also, lower contrast levels overall were noted for the LWIR projector because of ambient thermal radiation and reflections at the screen. The analysis of noise reveals that projector performance is better in MWIR than in LWIR as well. In the LWIR, the main drawback comes from the high spatial noise introduced by laser speckle. An alternative roughened screen was tested in an effort to reduce the influence of speckle over the image. This screen shows promising results. In both spectral bands, artifacts introduced in the image by diffraction patterns appear during alignment. Results, specifically in the MWIR, encourage further research and improvement of this low-cost projector for infrared simulation and training purposes.

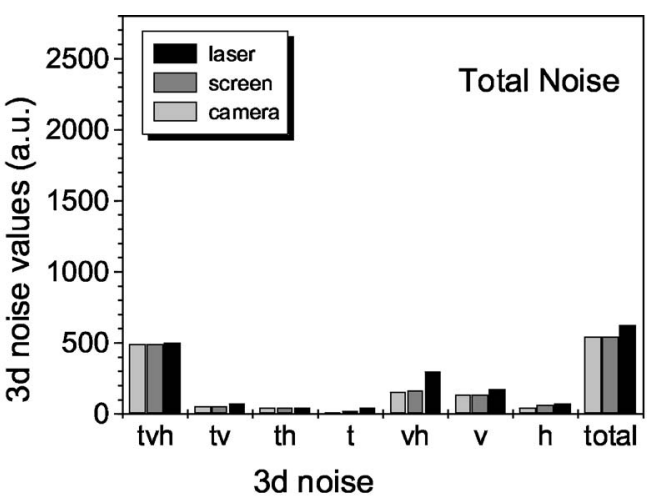

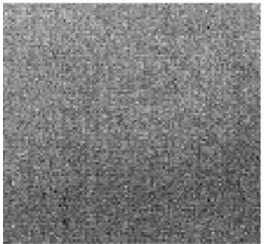

(a)

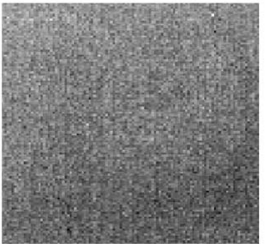

(b)

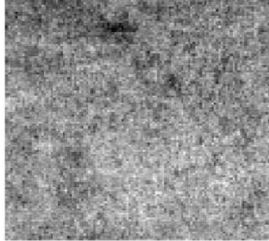

(c)
Fig. 8 3-D noise results for LWIR camera and the roughened metallic screen. Additional spatial noise with laser illumination is lower with the roughened surface relative to a sandblasted surface. The lower eigen-images are of the first principal component for (a) camera itself, (b) looking at the screen, and (c) illuminated with laser. Again, it is possible to see the influence of speckle, but its relevance is lower than when the sandblasted screen is used.

\section{References}

1. G. D. Boreman, M. Rahman, B. Monacelli, G. Zummo, D. Mullally, and T. Clarke, "Infrared targets for testing and training (IRT3)," Proc. SPIE 4717, 86-90 (2002).

2. D. Dudley, W. M. Duncan, and J. Slaughter, "Emerging digital micromirror device (DMD) applications," Proc. SPIE 4985, 14-25 (2003).

3. Optical Sciences Corporation, 4970 Corporate Drive, Suite $125 \mathrm{H}$, Huntsville, Alabama 35805. See http://www.opticalsciences.com.

4. K. Barnard, G. Boreman, and D. Pape, "Crosstalk model of a deformable-mirror infrared scene projector," Opt. Eng. 33(1), 140149 (1994).

5. G. D. Boreman, Modulation Transfer Function in Optical and Electro-Optical Systems, SPIE Press, Bellingham, WA (2001).

6. A. Daniels, G. Boreman, A. Ducharme, and E. Sapir, "Random transparency targets for modulation transfer function measurement in the visible and infrared," Opt. Eng. 34(3), 860-868 (1995).

7. D. N. Sitter, Jr., J. S. Goddard, and R. K. Ferrell, "Method for the measurement of the modulation transfer function of sampled imaging systems from bar-target patterns," Appl. Opt. 34, 746-751 (1995).

8. G. D. Boreman and S. Yang, "Modulation transfer function measurement using three- and four-bar targets," Appl. Opt. 34, 8050-8052 (1995).

9. W. R. Folks, D. Mullaly, G. Zummo, A. Weeks, and G. Boreman, "DMD-based infrared scene projection: A comparison of MWIR and LWIR modulation transfer function," Proc. SPIE 5408, 199-202 (2004).

10. G. C. Holst, Testing and Evaluation of Infrared Imaging Systems, Chap. 7, pp. 207-217, JCD Publishing, Bellingham (1998).

11. J. M. López-Alonso, J. Alda, and E. Bernabeu, "Principal components characterization of noise for infrared images," Appl. Opt. 41, 320-331 (2002).

12. J. M. López-Alonso and J. Alda, "Characterization of artifacts in fully-digital, image-acquisition systems. Application to web cameras," Opt. Eng. 43(1), 257-265 (2004).

13. R. Driggers, C. M. Webb, S. J. Pruchnic, C. E. Halford, and E. E. Burroughs, "Laboratory measurement of sampled infrared imaging system performance," Opt. Eng. 38(5), 852-861 (1999).

14. J. M. López-Alonso and J. Alda, "Operational parameterization of the $1 / \mathrm{f}$ noise of a sequence of frames by means of the principal compo- 
nents analysis in focal plane arrays," Opt. Eng. 42(7), 1915-1922 (2003).

15. D. B. Beasly, M. W. Bender, J. Crosby, T. Messer, and D. A. Saylor, "Advancements in the micromirror array projector technology," Proc. SPIE 5092, 71-82 (2003).

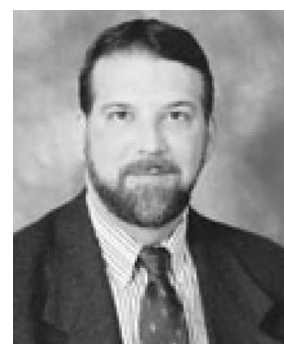

William R. Folks is a researcher at the University of Central Florida in Orlando. He has a BS from Eastern Michigan University and a $\mathrm{PhD}$ degree in physics from Kent State University. His research interests are in applied physics including liquid crystal displays, infrared optics, and optical engineering.

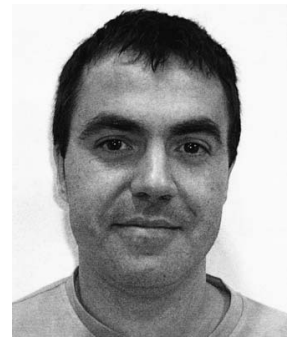

José Manuel López-Alonso has directed the Thermovision Laboratory section at Centro de Investigaciónn y Desarrollo de la Armada since 1998, developing figures of merit for the characterization of IR and visible imagers. He received his Lic degree in physics from the University Complutense of Madrid, where he is currently working in the optics department as associate professor. Since 1998 he has been the Spanish delegate for group TG12 of the North Atlantic Treaty Organization devoted to the characterization of thermal imagers. He is also involved in the development of European EUCLID programs on electro-optics topics.

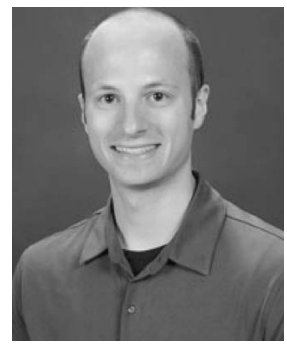

Brian Monacelli received the BS degree in applied optics at Rose-Hulman Institute of Technology, Terre Haute, Indiana, in 2000 and the MS degree in optics from the Institute of Optics, University of Rochester, New York, in 2001 . He is currently a graduate research and teaching assistant while working toward the PhD in optics at the University of Central Florida. His research interests include infrared frequency selective surfaces, uncooled antenna-coupled infrared detectors, and infrared system design. He has worked as a research scientist with Rocketdyne Boeing Lasers (Electro-Optics Systems Division), Silicon Valley Group (Lithography Division), and NSWCCrane. He is a student member of SPIE, OSA, and IEEE. He currently serves as President of the UCF Student Chapter of the Optical Society of America.

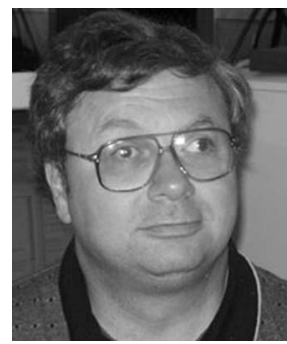

Arthur Weeks received his $\mathrm{PhD}$ degree in electrical engineering from the University of Central Florida in Orlando in 1987. After completion of his PhD, he spent one year at the Royal Signals and Radar Establishment in Malvern, England, studying laser beam propagation. He left the position of Vice President of Corporate Technology at Invivo Research Incorporated to join the electrical engineering department at the University of Central Florida as an associate professor. His interests include color image processing techniques, the reduc- tion of noise within images using adaptive nonlinear filters, and the use of artificial neural networks in pattern recognition. He is a member of the IEEE, SPIE, and Tau Beta Pi.

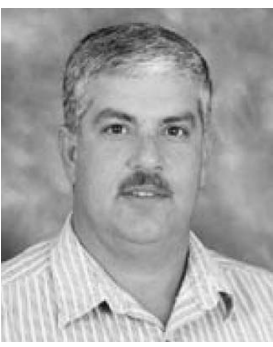

Guy Zummo is a staff engineer at the College of Optics and Photonics at the University of Central Florida in Orlando.

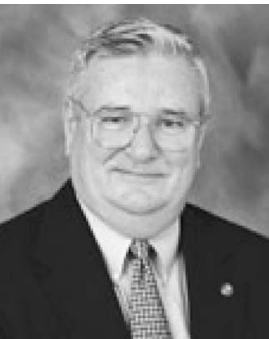

Daniel E. Mullally is currently employed in new business development for the IR Systems Laboratory, CREOL, College of Optics and Photonics, at the University of Central Florida. He earned his BS in business administration from Western Carolina University in 1957 and his MA in human resource management from Pepperdine University in 1983. He is a retired U.S. Marine Corps Infantry Officer who served in the USMC from 1957 to 1977.

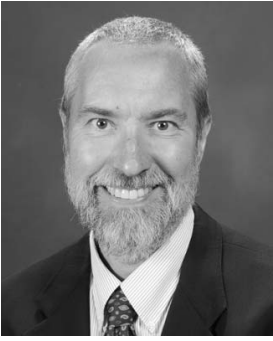

Glenn D. Boreman is Trustee Chair Professor of Optics, Electrical Engineering, and Physics at the College of Optics and Photonics (CREOL) at the University of Central Florida. He received a BS from the Institute of Optics, University of Rochester, and a $\mathrm{PhD}$ from the Optical Sciences Center, University of Arizona. He has been a visiting scholar at Imperial College in London, the Swiss Federal Institute of Technology (ETH) in Zürich, and the Defense Research Agency (FOI) in Linköping, Sweden. He currently serves as the editor-in-chief of OSA's journal Applied Optics, and is a past member of the SPIE Board of Directors. He is coauthor of the graduate textbook Infrared Detectors and Systems (Wiley-Interscience, 1996), author of Modulation Transfer Function in Optical and Electro-Optical Systems (SPIE, 2001), and Basic Electro-Optics for Electrical Engineers (SPIE, 1998). He has published more than 100 articles in the areas of infrared detector and focal-plane analysis, optics of random media, infrared scene projection, and transferfunction techniques. He is a Fellow of SPIE and OSA. He and two of his students received the 1995 Kingslake Medal from SPIE. 